\title{
Assessing Performance of Internet of Things-based Mobile Crowdsensing Systems for Sensing as a Service Applications in Smart Cities
}

\author{
Andrea Capponi, Claudio Fiandrino, Christian Franck, Ulrich Sorger, Dzmitry Kliazovich and Pascal Bouvry \\ Faculty of Science, Technology and Communication \\ University of Luxembourg \\ \{firstname.lastname\}@uni.lu
}

\begin{abstract}
The Internet of Things (IoT) paradigm makes the Internet more pervasive. IoT devices are objects equipped with computing, storage and sensing capabilities and they are interconnected with communication technologies. Smart cities exploit the most advanced information technologies to improve public services. For being effective, smart cities require a massive amount of data, typically gathered from sensors. The application of the IoT paradigm to smart cities is an excellent solution to build sustainable Information and Communication Technology (ICT) platforms and to produce a large amount of data following Sensing as a Service $\left(\mathrm{S}^{2}\right.$ aaS) business models. Having citizens involved in the process through mobile crowdsensing (MCS) techniques unleashes potential benefits as MCS augments the capabilities of existing sensing platforms. To this date, it remains an open challenge to quantify the costs the users sustain to contribute data with IoT devices such as the energy from the batteries and the amount of data generated at city-level. In this paper, we analyze existing solutions, we provide guidelines to design a large-scale urban level simulator and we present preliminary results from a prototype.
\end{abstract}

Keywords-Mobile crowdsensing, IoT, smart cities, sensing as a service

\section{INTRODUCTION}

In IoT, everyday life objects are "smart", i.e, they can communicate one with each other and with users to enable pervasive and ubiquitous computing [1]. IoT devices are objects uniquely identifiable and are equipped with communication, computing, storage and sensing capabilities. Applying the IoT paradigm to urban scenarios is of special interest to support the smart cities vision [2], [3]. Smart cities aim at using ICT solutions to improve the quality of life of citizens by provisioning innovative solutions for public services such as healthcare, public safety and smart transportation among others [2], [4]. The IoT paradigm is the candidate building block to develop sustainable ICT platforms for smart cities. Including citizens in the loop with crowdsensing techniques augments capabilities of existing infrastructures without additional costs and is proved to be a win-win strategy for urban applications [5].

MCS is an appealing paradigm for sensing and has gained a growing attention in the last years. Fig. 1 illustrates the main elements of a MCS system. Source of data of MCS systems are IoT devices, smartphones, tablets and wearable devices that are becoming widespread and popular [6]. All these devices are equipped with sensing capabilities and utilized by large number of users, the crowd. Unlike fixed sensor networks, MCS systems enable virtually unlimited

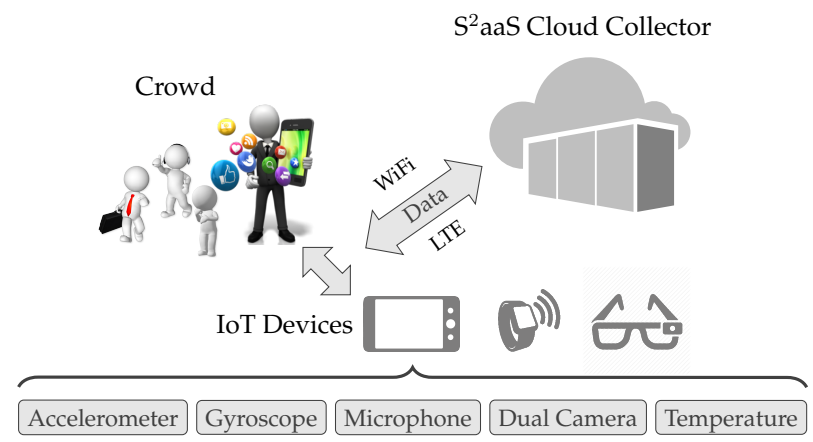

Figure 1. Cloud-based MCS system

sensing possibilities. First, the devices have several types of sensors and are periodically recharged by users. Second, intelligence of human participants and mobility augment context-awareness and coverage. Companies have a growing interests in MCS. Google, for example, uses crowd-sourced information of smartphones' locations to offer real-time view of congested traffic roads and has recently released a new application, called Science Journal, which allows to gather and visualize data from smartphones' sensors [7]. Pokemon Go is a extremely popular MCS framework for collecting data like user location and movements. In the US, $10 \mathrm{M}$ users downloaded the application in two weeks.

Sensing as a Service ( $\mathrm{S}^{2}$ aaS $)$ makes available to the public data collected from sensors. Consequently, companies have no longer the need to acquire an infrastructure to perform a sensing campaign. IoT and MCS are key enablers in the $S^{2}$ aaS model, which in turn is envisioned to play are indispensable role in smart cities. Efficiency of $\mathrm{S}^{2}$ aaS models is defined in terms of the revenues obtained selling data and the costs. In $\mathrm{S}^{2}$ aaS, the organizer of a sensing campaign, such as a government agency, an academic institution or business corporation, sustains costs to recruit and compensate users for their involvement [8]. Also the users sustain costs while contributing data. These costs are the energy spent from the batteries for sensing and reporting data and, eventually, the data subscription plan if cellular connectivity is used for reporting.

As for proper operation MCS systems require the contribution from a large number of participants, the development of real testbeds is not feasible. Therefore, simulations are the candidate tool to assess the costs and understand the performance of MCS systems. In this paper, we illustrate 
the key principles for the design of a MCS simulator, the fundamental key performance indicators (KPIs) to be assessed and we present results obtained from a prototype. The most important features considered in the design are: i) realistic urban environment, ii) large scale scenarios, i.e., a large number of participants and prolonged time duration to mimic realistic sensing campaigns.

\section{BACKGROUND}

This section reviews existing works in the field of performance evaluation of MCS systems through simulations.

Tanas et al. propose to exploit Network Simulator 3 (NS3) for crowdsensing simulations [9]. The objective is to assess the performance of a crowdsensing network taking into account the mobility properties of the nodes together with the wireless interface in ad-hoc network mode. Furthermore, the authors present a case study about how participants could report incidents in the public rail transport. NS-3 provides highly accurate estimations of network properties. However, having detailed information on communication properties comes with the cost of losing scalability. First, it is not possible to simulate tens of thousands of users contributing data. Second, the granularity of the duration of NS-3 simulations is typically in the order of minutes. Indeed, the objective is to capture specific behaviors such as the changes of the TCP congestion window. However, the duration of real sensing campaigns is typically in the order of hours or days.

In [10], Farkas and Lendák present a simulation environment developed to investigate performance of crowdsensing applications in an urban parking scenario. Although the application domain is only parking-based, the authors claim that the proposed solution can be applied to other crowdsensing scenarios. However, the scenario considers only drivers as type of users and users travel from one parking spot to another one. The authors consider humans as sensors that trigger parking events. However, to be widely applicable, a crowdsensing simulator has to take into account data generated from mobile and IoT devices' sensors carried by human individuals.

Mehdi et al. propose CupCarbon [11], which is a discreteevent wireless sensor network (WSN) simulator for IoT and smart cities. One of the major strengths is the possibility to model and simulate WSN on realistic urban environments through OpenStreetMap. To set up the simulation, the users have to deploy on the map the various sensors and the nodes such as mobile users, gas and media sensors and base stations. The approach is not intended for crowdsensing scenarios with thousands of users.

\section{DESIGN PRINCIPLES AND KPI FOR MOBILE CROWDSENSING Simulators}

The objective of this section is to outline the design principles and the main key performance indicators MCS simulators should include.

\section{A. Design Principles}

To design a novel MCS simulator, the main aspects to consider are the scalability, the implementation in a realistic urban environment, the user mobility and the communication technologies.

Scalability: For proper operation, MCS systems require a large number of participants. Hence MCS simulators should be designed to host in the order of tens of thousands participants moving in a wide geographical space. Each user can potentially own several IoT and mobile devices, each of them is a potential data contributor. Time dimension is also important. The duration of a sensing campaign ranges from hours to days and a simulator should address this challenge efficiently. For instance, let us consider 10000 users producing data with an average of only 30 minutes per day. Each user delivers 12 bits long samples of the accelerometer working at $50 \mathrm{~Hz}$ frequency. The total amount of generated data is $1.35 \mathrm{~GB}$. Considering prolonged duration of user contribution and additional sensors would considerably augment the figure.

Realistic urban environment: Similarly to CupCarbon, MCS simulators should rely on realistic urban environments for several reasons. First, exploiting realistic layouts of urban environments makes the simulator flexible and easy to be adopted in any city. Second, it allows to perform analysis providing meaningful insights to the municipality to understand the feasibility and the potentiality of the proposed MCS solution. Simulations over a grid or a square area as abstraction levels lower the complexity, but do not allow to take into account important features such as movements in real streets and physical obstacles like buildings.

User mobility: Human mobility is defined as sequences of spatiotemporal user movements. Understanding human mobility urban environments is crucial to design mobility patterns that meet social behaviors and scale to the requirements of modern smart cities [12].

Communication technologies: IoT and mobile devices are equipped with several communication technologies, including 3G/LTE, WiFi and Bluetooth. Each communication technology drains battery of the devices differently and can have associated costs (e.g., users have a limited monthly plan).

\section{B. Types of KPI}

This subsection details important types of KPI that MCS simulators should assess, including data generation and cost evaluation.

Data Generation: Sensors work with different sampling frequency and sample size. After data collection, mobile devices deliver samples to a central collector using different communication technologies. In $\mathrm{S}^{2}$ aaS models, revenues are proportional to the amount of generated data. Therefore, it is 
Table I

SENSOR AND COMMUNICATION EQUIPMENT PARAMETERS USED FOR PERFORMANCE EVALUATION

\begin{tabular}{llrl}
\hline SENSOR & PARAMETER & VALUE & UNIT \\
\hline Accelerometer & Sample rate & 50 & $\mathrm{~Hz}$ \\
& Sample size & 12 & $\mathrm{Bits}$ \\
& Current & 35 & $\mu \mathrm{A}$ \\
\hline Temperature & Sample rate & 182 & $\mathrm{~Hz}$ \\
& Sample size & 16 & $\mathrm{Bits}$ \\
& Current & 182 & $\mu \mathrm{A}$ \\
\hline Pressure & Sample rate & 157 & $\mathrm{~Hz}$ \\
& Sample size & 16 & $\mathrm{Bits}$ \\
& Current & 423.9 & $\mu \mathrm{A}$ \\
\hline
\end{tabular}

\begin{tabular}{lrll}
\hline SYMBOL & VALUE & UNIT & DESCRIPTION \\
\hline$\rho_{i d}$ & 3.68 & $\mathrm{~W}$ & Energy in idle mode \\
$\rho_{t x}$ & 0.37 & $\mathrm{~W}$ & Transmission power \\
$\rho_{r x}$ & 0.31 & $\mathrm{~W}$ & Reception power \\
$\lambda_{g}$ & 1000 & $\mathrm{fps}$ & Rate of generation of packets \\
$\gamma_{x g}$ & $0.11 \cdot 10^{-3}$ & $\mathrm{~J}$ & Energy cost to elaborate a generated packet \\
\hline
\end{tabular}

(b) Communication Equipment

(a) Sensor Equipment

important to assess data generation KPI such as the amount of data generated in a given time window, or per area.

Cost evaluation: Nowadays energy consumption is one of the most important and challenging issues worldwide. In MCS, energy is consumed to perform sensing and reporting. The energy spent per sensing is typically proportional to the sampling frequency of the sensor. The energy spent for communications depends on the technology used. Rapid battery drain due to MCS-related applications can lower user participation.

\section{PRELIMINARY RESUltS}

Following the design principles illustrated in Section III, this section presents a prototype for a MCS simulator and illustrates preliminary results. The experiments aim at assessing the amount of data gathered and the energy consumption of the devices for sensing and reporting.

\section{A. Simulation scenario}

The developed prototype is a discrete-event simulator supporting pedestrian mobility. Users move in the city center of Luxembourg, which covers an area of $1.11 \mathrm{~km}^{2}$. To obtain information about the streets of the city, the simulator exploits a crowdsourced application providing free access to streetlevel maps ${ }^{1}$ in form of a set of coordinates $\mathcal{C}$ containing $<$ latitude, longitude, altitude $>$. The original locations of the users randomly assigned form the set of coordinates $\mathcal{C}$. The number of participants is set to 20000 , which corresponds to approximately $20 \%$ of the population of Luxembourg (115 200 inhabitants as of end 2015). The start time of the walk is uniformly distributed between 8:00 AM and 1:30 PM. Each participant has only one mobile device and walks for a period of time that is uniformly distributed between 10 and 30 minutes. Users move with an average velocity speed uniformly distributed between 1 and $1.5 \mathrm{~m} / \mathrm{s}$. The participants collect data and deliver it to the collector while walking. Once the period of walking ends, they stop moving and contributing. Consequently, users generate information for a little period of time along the day, which allows to study the system performance under a worst case scenario.

\footnotetext{
${ }^{1}$ DigiPoint: http://www.zonums.com/gmaps/digipoint.php
}

Data generation takes into account sensors commonly available in current IoT and mobile devices. Table I presents detailed information on sensors and communication parameters. Specifically, the sensing equipment consists of the FXOS8700CQ 3-axis linear accelerometer from Freescale Semiconductor [13] and the BMP280 from Bosch [14], which is a digital pressure and temperature sensor. Data delivery occurs using WiFi with the precise location of WiFi hotspots in form of $<$ latitude, longitude $>^{2}$. Without loss of generality, considering only WiFi communication technology simplifies the understanding of data collection and energy consumption.

The energy $E$ spent during the transmission time $\tau_{t x}$ is defined as:

$$
E=\int_{0}^{\tau_{t x}} P_{t x} \mathrm{~d} t,
$$

where $P_{t x}$ is the power consumed for transmissions of WiFi packets generated at rate $\lambda_{g}[15]$ :

$$
P_{t x}=\rho_{i d}+\rho_{t x} \cdot \tau_{t x}+\gamma_{x g} \cdot \lambda_{g}
$$

\section{B. Simulation results}

The Section illustrates preliminary results aiming at assessing the cost the users sustain in terms of energy consumption.

Fig. 2 shows the distribution of user energy consumption due to sensing and reporting. Currently the battery capacity of mobile devices is quantified in terms of current drain $(\mathrm{mAh})$, therefore in the evaluation we assess the current drain of the three sensors per user. As data is immediately delivered to the collector with $\mathrm{WiFi}$ after being produced, the amount of collected data impacts proportionally on sensing and communication costs. The profile follows a normal distribution as the users that are moving for prolonged time periods contribute more data than users walking for short periods. On average, each user spends for sensing 374.617 $\mu$ Ah (see Fig. 2(a)). The few top contributors experience a consumption that is approximately double compared to the average. Considering that in current smartphones the battery capacity is approximately $2000 \mathrm{mAh}$, we can conclude that the energy spent for sensing is negligible when compared to the energy spent for communications

\footnotetext{
${ }^{2}$ Online: https://www.hotcity.lu/en/laptop/www/About/Wi-Fi-coverage
} 


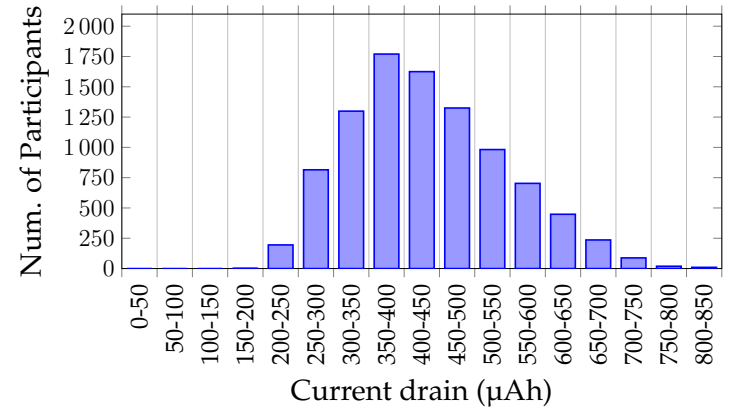

(a) Sensing Cost

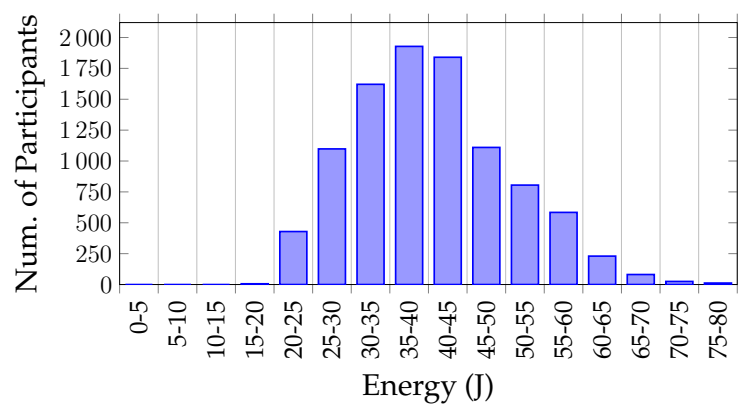

(b) Communication Cost

Figure 2. Energy spent for sensing and communication

(see Fig. 2(b)). To reduce energy spent for communications, one possibility is to defer data transmission and not transmit samples continuously.

\section{CONClusion ANd Future Research Directions}

This paper proposes design principles and KPI for the development of simulators and performance evaluation of MCS systems. We first analyze existing simulators in the field and we pose the basis to develop a novel simulator illustrating a prototype. The objective is to provide a simulation environment to evaluate and assess MCS systems for different applications and services in any real urban environments. According to the design principles presented, the prototype we propose takes into account realistic urban scenarios, different communication technologies, sensors commonly available in mobile devices and supports pedestrian mobility. For performance evaluation, we present preliminary results aiming to assess the energy cost the users sustain to collect and report sensing data.

For future work we plan to further develop the prototype in order to assess and compare the performance of MCS systems in any real city. The simulator is projected to include different types of citizen mobility (e.g., pedestrian, by car or public transports) and the most widespread communication technologies like cellular and Bluetooth in addition to WiFi. Furthermore, the prototype will include a graphic and userfriendly interface.

\section{ACKNOWLEDGMENT}

This material is based upon work supported by the National Research Fund, Luxembourg in the framework of ECOCLOUD and iShOP projects.

\section{REFERENCES}

[1] A. Al-Fuqaha, M. Guizani, M. Mohammadi, M. Aledhari, and M. Ayyash, "Internet of things: A survey on enabling technologies, protocols, and applications," IEEE Communications Surveys Tutorials, vol. 17, no. 4, pp. 2347-2376, 2015.

[2] A. Zanella, N. Bui, A. Castellani, L. Vangelista, and M. Zorzi, "Internet of things for smart cities," IEEE Internet of Things Journal, vol. 1, no. 1, pp. 22-32, Feb 2014.

[3] C. Perera, A. Zaslavsky, P. Christen, and D. Georgakopoulos, "Sensing as a service model for smart cities supported by Internet of Things," Transactions on Emerging Telecommunications Technologies, vol. 25, no. 1, pp. 81-93, 2014.
[4] M. Pouryazdan, C. Fiandrino, B. Kantarci, D. Kliazovich, T. Soyata, and P. Bouvry, "Game-Theoretic Recruitment of Sensing Service Providers for Trustworthy Cloud-Centric Internet-of-Things (IoT) Applications," in 5th Workshop on Cloud Computing Systems, Networks, and Applications (CCSNA) in conjunction with IEEE GLOBECOM, Dec. 2016.

[5] J. G. P. Rodrigues, A. Aguiar, and J. Barros, "Sensemycity: Crowdsourcing an urban sensor," CoRR, 2014.

[6] C. Ragona, F. Granelli, C. Fiandrino, D. Kliazovich, and P. Bouvry, "Energy-efficient computation offloading for wearable devices and smartphones in mobile cloud computing," in IEEE Global Communications Conference (GLOBECOM), Dec 2015, pp. 1-6.

[7] Google Inc., "Google science journal," 2016. [Online]. Available: https://makingscience.withgoogle.com/science-journal/

[8] C. Fiandrino, B. Kantarci, F. Anjomshoa, D. Kliazovich, P. Bouvry, and J. Matthews, "Sociability-Driven user recruitment in mobile crowdsensing Internet of Things Platforms," in IEEE Global Communications Conference (GLOBECOM), Dec 2016.

[9] C. Tanas and J. Herrera-Joancomartí, "Crowdsensing simulation using ns-3," Citizen in Sensor Networks: Second International Workshop, CitiSens 2013, pp. 47-58, 2014, Springer International Publishing.

[10] K. Farkas and I. Lendák, "Simulation environment for investigating crowd-sensing based urban parking," in International Conference on Models and Technologies for Intelligent Transportation Systems (MT-ITS), June 2015, pp. 320-327.

[11] K. Mehdi, M. Lounis, A. Bounceur, and T. Kechadi, "Cupcarbon: A multi-agent and discrete event wireless sensor network design and simulation tool," in 7th International ICST Conference on Simulation Tools and Techniques, ser. SIMUTools '14, 2014, pp. 126-131.

[12] K. K. Jahromi, M. Zignani, S. Gaito, and G. P. Rossi, "Simulating human mobility patterns in urban areas," Simulation Modelling Practice and Theory, vol. 62, pp. 137 - 156, 2016.

[13] "FXOS8700CQ: Digital Sensor - 3D Accelerometer + 3D Magnetometer," http://cache.nxp.com/files/sensors/doc/data_ sheet/FXOS8700CQ.pdf?pspll=1, 2015.

[14] "BMP280, Barometric Pressure Sensors," https://www. bosch-sensortec.com/bst/products/all_products/bmp280, 2015.

[15] A. Garcia-Saavedra, P. Serrano, A. Banchs, and G. Bianchi, "Energy consumption anatomy of 802.11 devices and its implication on modeling and design," in 8th International Conference on Emerging Networking Experiments and Technologies, ser. CoNEXT' '12, 2012, pp. 169-180. 